\title{
An Exploration of Issues and Limitations in Current Methods of TOPSIS and Fuzzy TOPSIS
}

\author{
Elissa Nadia Madi, Jonathan M. Garibaldi, Christian Wagner \\ Lab for Uncertainty in Data and Decision Making (LUCID) and IMA Research Group, \\ School of Computer Science, University of Nottingham, UK \\ \{elissa.madi,jon.garibaldi,christian.wagner\}@nottingham.ac.uk
}

\begin{abstract}
Multi Criteria Decision Making is a challenging but vital process for organizations. One of the best-known techniques to support Multi-Criteria Decision Making is the 'Technique for Order Preference by Similarity to Ideal Solution' (TOPSIS) approach. In recent years, a variety of extensions, including fuzzy extensions of TOPSIS have been proposed. Besides the many variations of standard TOPSIS, one family of extensions employing fuzzy sets is referred to as fuzzy TOPSIS (FTOPSIS). One challenge that has arisen is that it is not straightforward to choose between the multiple variants of TOPSIS existing today. Previously, none of the papers that have compared the key differences between standard and fuzzy TOPSIS have fully explored each of the step-wise stages. In this paper, we now provide a detailed comparison of these key stages in a systematic stepwise manner, clearly highlighting differences. We also identify and discuss the limitations, issues and challenges which exist in the present FTOPSIS method. The crucial and main issues are identified as relating to concepts of reliability, truth and meaning. Having identified these conceptual issues, we then go on to highlight what we argue to be the main issue, that of reliability, to discuss further. We proceed to present a potential solution and propose a framework to address the issue. This study will provide guidelines to researchers in this field and to provide potential pathways to further solutions, which have the capacity to advance the area of FTOPSIS as a whole.
\end{abstract}

\section{INTRODUCTION}

Decision making is an important process for organizations. Common practice involves evaluation of prioritized alternatives based on a given set of criteria. These criteria conflict with each other and commonly no solution can satisfy all criteria simultaneously. This problem is known as Multi Criteria Decision Making (MCDM) or Multi Criteria Decision Analysis (MCDA) problem. One of the well-known techniques in MCDM is the 'Technique for Order Preference by Similarity to Ideal Solution' (TOPSIS) which was introduced by Hwang and Yoon in 1981 [1]. However, this technique uses crisp information which is impractical in many real world situations because decision makers usually express opinions in natural language such as Poor and Good. Information in the form of natural language, i.e. words, in turn is characterized by fuzziness and uncertainty (i.e. 'what is the meaning of poor'). This uncertainty can be a challenge for decision makers. Zadeh [2] introduced the concept of fuzzy sets, which enables systematic reasoning with imprecise and fuzzy information by using fuzzy sets to represent linguistic terms numerically to then handle uncertain human judgement.
An impressive variety of fuzzy decision making applications have been developed in the last two decades. Behzadian et al. [3] reviewed in particular applications of TOPSIS and found that using fuzzy sets seems to be the most commonly used method in TOPSIS. Specifically, while the classical TOPSIS method assumes that alternative ratings and criteria weights are crisp numbers, more than half of the TOPSIS publications $(52.2 \%)$ utilized linguistic variables and fuzzy sets to handle problems with imprecise information. They also found that most TOPSIS publications (76 papers) were related to group decision making issues.

An initial extension of the TOPSIS method for group decision-making under fuzzy environment by Chen [4] in 2000 can be considered as one of the early works in this area. Based on the work, many researchers started to explore and enhance this method. Various types of enhancements were made. For example, some proposed to use different types of fuzzy information (e.g., type-1 fuzzy sets [4], type-2 sets [5], intuitionistic sets [6], interval valued sets [7], etc.) in order to handle impreciseness of information in the problem.

Despite many variations of fuzzy TOPSIS (FTOPSIS) methods, none of the papers explaining the details of the key stages of standard and fuzzy TOPSIS are based on stepwise procedure. Furthermore, most papers do not highlight the differences between these two methods in clear detail. Thus, it is difficult to determine the limitations of the various methods. The process of identifying the limitations is crucial in order to provide meaningful justification or logical reason why such method should be chosen, as well as to provide confidence in the recommended decision.

In this paper, we will provide the detail about the key stages involved in both standard TOPSIS and FTOPSIS in a systematic stepwise manner. Further, we will highlight clearly which stage is different between TOPSIS and FTOPSIS method. In order to illustrate how FTOPSIS method works, we provide a real-world numerical example taken from [4]. Then, we identify and discuss the limitations, issues and challenges in the existing FTOPSIS method. The crucial and main issues are identified as relating to concepts of reliability, truth and meaning. Here, we only highlight one main issue, namely reliability, to discuss further in fuzzy TOPSIS problem. We proceed to discuss a potential solution and proposed a framework to addressed the issue.

The rest of this paper is organized as follows. In Section 
II-A, a detailed discussion about the key stages in standard TOPSIS is provided. Following on from this discussion, in Section III, we proceed to provide the details of key stages in conventional FTOPSIS as well as the numerical example to show how these stages work and provide discussion about basic extensions made from few researchers. Then, we provide a detailed discussion about current limitations, challenges and issues in existing FTOPSIS methods and discuss it in detail in Section IV before introducing a potential solution in Section V. Finally, Section VI provides conclusions and details of future work.

\section{BACKGROUND}

\section{A. TOPSIS}

In MCDM techniques, there are two basic approaches: multiple attribute decision making (MADM) and multiple objective decision making (MODM) [8]. TOPSIS fall in the MADM category which refers to making selection among some courses of action in the presence of multiple, usually conflicting, attributes. It is assumed that there exist a limited number of predetermined alternatives in such problems. The standard TOPSIS method principle is to choose alternatives that simultaneously have the shortest distance from the positive ideal solution (hypothetical best alternative) and the farthest distance from the negative-ideal solution (hypothetical worst alternative). The positive ideal solution maximizes the benefit criteria and minimizes the cost criteria, whereas the negative ideal solution maximizes the cost criteria and minimizes the benefit criteria. On the other hand, MODM is the technique that has infinite number of alternatives and typically no given alternatives. Only TOPSIS method is considered in this paper.

A standard MADM problem can be concisely expressed in decision matrix format as

$$
\begin{gathered}
\mathbf{D}=\begin{array}{l}
C_{1} \\
A_{1} \\
A_{2} \\
\vdots \\
A_{m}
\end{array}\left[\begin{array}{cccc}
x_{11} & x_{12} & \cdots & x_{1 n} \\
x_{21} & x_{22} & \cdots & x_{2 n} \\
\vdots & \vdots & \ddots & \vdots \\
x_{m 1} & x_{m 2} & \cdots & x_{m n}
\end{array}\right], \\
\mathbf{W}=\left[\begin{array}{llll}
w_{1} & w_{2} & \cdots & w_{n}
\end{array}\right],
\end{gathered}
$$

where $A_{1}, A_{2}, \cdots, A_{m}$ are possible alternatives among which DMs have to choose, $C_{1}, C_{2}, \cdots, C_{n}$ are criteria/attributes with which measured, $x_{i j}$ is the rating of alternative $A_{i}$ with respect to criterion $C_{j}$ and $w_{j}$ is the weight of criterion $C_{j}$.

In standard TOPSIS, it is assumed that each attribute in the decision matrix, D takes either monotonically increasing or monotonically decreasing utility. In other words, the larger the attribute outcomes is, the greater the preference for the 'benefit' criteria and the less the preference for the 'cost' criteria. Furthermore, any outcome which is expressed in a nonnumerical way should be quantified through the appropriate scaling technique, for example, using a rating scale on certain points on ' $0-10$ ' or ' $0-100$ ' by a group of DMs. Since all criteria cannot be assumed to be of equal importance, the method receives a set of weights from the decision makers and can be expressed as in matrix $\mathbf{W}$ using the weight of importance scale.

Figure 1 shows the stepwise TOPSIS procedure from Hwang and Yoon [1]. After forming an initial decision matrix, D, the procedure starts by normalizing the decision matrix. This step aims to transform the various attribute dimensions into non-dimensional attributes, which allows comparison across the attributes. There are many ways to normalize it as describe by Chen and Hwang [9].

Here, the normalization step is made by taking the outcome of each criterion divided by the norm of the total outcome vector of the criterion at hand as shown in Figure 1. For step 2, a set of weight expressed in matrix $\mathbf{W}$ given by DMs are accommodated to the decision matrix, $\mathbf{D}$ by multiplying each column of the matrix $\mathbf{D}$ (i.e., $r_{i j}$ value) with its associated weight $w_{j}$. Then, in Step 3, two artificial alternatives known as Positive Ideal Solution (PIS) and Negative Ideal Solution (NIS) are defined. $A^{*}$ and $A^{-}$indicate the most preferable alternative (PIS) and the least preferable alternative (NIS), respectively. In this step, $J$ associated with benefit criteria while $J^{\prime}$ is cost criteria. Step 4 is a calculation separation between each alternative from the ideal one by the $n$-dimensional Euclidean distance. Then, in the final step, the relative closeness $\left(C C_{i}\right)$ of alternative $i$ th $\left(A_{i}\right)$ with respect to $A^{*}$ is calculated. It is clear that $C C_{i}=1$ if $A_{i}=A^{*}$ and $C C_{i}=0$ if $A_{i}=A^{-}$. An alternative $A_{i}$ is closer to $A^{*}$ as $C C_{i}$ approaches 1 . Next, an alternative can now be ranked according to the descending order of $C C_{i}$. Overall, TOPSIS makes full use of attribute information, provides a cardinal ranking of alternatives, and does not require attribute preferences to be independent [9]. In the next section, we provides the detail of TOPSIS extension using fuzzy sets.

\section{FUZZY TOPSIS (FTOPSIS)}

Zadeh [2] introduced fuzzy set theory to deal with uncertainty and impreciseness in real-world information. The success of such theory in solving various application has motivated researchers to integrate it in decision making technique. In standard TOPSIS method [1], the ratings and the weights of criteria are known precisely and it used crisp data to model real-world situations. However, in most situations, it is impractical to use crisp data to model such situations. For example, human judgements or preferences are often vague and cannot estimate such preferences in exact numerical form. Thus, fuzzy sets can be used to express preferences using linguistic variables.

The extension of TOPSIS by Chen [4] can be assumed as one of the pioneer works in this field. In this work, fuzzy sets were used to present the linguistic term when decision makers were asked to give rating of each alternative. Based on this, many researchers started to explore and enhance this initial Fuzzy TOPSIS (FTOPSIS) method. In this section, we provide the stepwise procedure of the original FTOPSIS method taken from Chen [4]. We also discuss which step has been enhanced and the differences between the standard TOPSIS from Hwang and Yoon [1] with the FTOPSIS method from Chen [4]. 


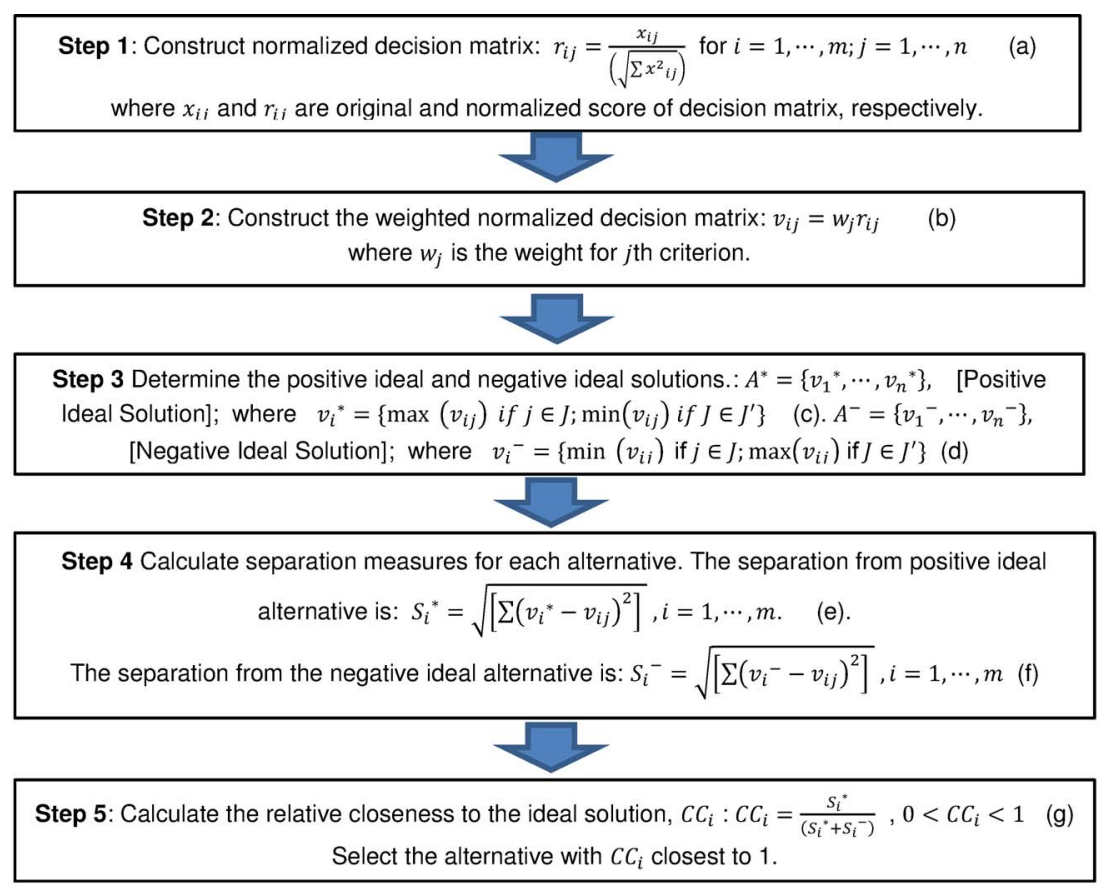

Fig. 1. Stepwise procedure of TOPSIS [9]

In FTOPSIS, a special type of fuzzy set, namely a fuzzy number, is used to represent the human preferences. A fuzzy number is a fuzzy subset in the universe of discourse $X$ that is both convex and normal. There are different types of fuzzy numbers based on applications or situations. The triangular fuzzy number (TFN) is most widely used in decision making because of its intuitive membership functions and computational simplicity [10] [11]. A triangular fuzzy number can be defined as a triplet $(l, m, u)$. The parameters $l, m$ and $u$, respectively, specify the smallest possible value, the peak value and the largest possible value of the domain of the membership function.

In the beginning of this method, the same matrix forms are used to present the rating of alternatives, namely the decision matrix $\mathbf{D}$ and $\mathbf{W}$ for the weight of each criterion (please refer Section II-A). However, instead of using crisp numbers in the rating value of $x_{i j}$, the FTOPSIS method uses fuzzy numbers (FN) to represent them. For example, for rating Good, the associated triangular FN is $(7,9,10)$. In contrast, in standard TOPSIS, the value of Good might be the crisp value 9 .

Assume that a decision group consist of $k$ decision makers, then the average weights of criteria and ratings of alternatives given by $k$ decision makers can be computed as:

$$
\begin{gathered}
\tilde{w}_{j}=\frac{1}{k}\left[\tilde{w}_{j}^{1}+\tilde{w}_{j}^{2}+\cdots+\tilde{w}_{j}^{k}\right] \\
\tilde{x}_{j}=\frac{1}{k}\left[\tilde{x}_{j}^{1}+\tilde{x}_{j}^{2}+\cdots+\tilde{x}_{j}^{k}\right]
\end{gathered}
$$

where $\tilde{w}_{j}$ and $\tilde{x}_{j}$ are the importance weights of criteria and the ratings of alternatives given by $k$ th decision maker. The resulting values of $\tilde{w}_{j}$ and $\tilde{x}_{j}$ are expressed in decision matrix $\mathbf{W}$ and $\mathbf{D}$, respectively. The rating and the weights are described as triangular fuzzy number which indicate $\tilde{x}_{j}=\left(a_{i j}, b_{i j}, c_{i j}\right)$ and $\tilde{w}_{j}=\left(w_{j 1}, w_{j 2}, w_{j 3}\right)$. Then, the stepwise procedure of FTOPSIS is summarized and shown in Figure 2.

Based on Figure 2, the calculation in Step 1 is slightly different compared to Step 1 in Figure 1. The reason is that different type of information has been used to express human judgement given by decision makers. In FTOPSIS method, triangular fuzzy numbers are used, as opposed to crisp numbers in standard TOPSIS method. In Step 1, the fuzzy number for its associated membership function is expressed as $\left(a_{i j}, b_{i j}, c_{i j}\right)$, where $a, b$ and $c$ are the smallest possible value, the peak value and the largest possible value, respectively. The normalization step is needed to transform various criteria scales into a single comparable scale, and to preserve the property of normalized triangular fuzzy number belonging to $[0,1]$. Then, Step 2 is the same as the standard TOPSIS used to compute the weighted normalized decision matrix, as shown in Step 2 of Figure 2. In Step 3, the fuzzy PIS and fuzzy NIS values are determined as $(1,1,1)$ and $(0,0,0)$, respectively. Obviously, both values are expressed in triangular fuzzy number form. This means the FTOPSIS method considers the ideal solution in the perfect condition where 1 means the perfect good point and 0 the perfect least point. In Step 4, standard TOPSIS and FTOPSIS method has the same objective in which the separation measure is calculated for each alternative from both ideal solutions. In FTOPSIS, the separation measure is based on a vertex method in which each value in TFN is considered simultaneously, as shown in Step 4 of Figure 2. Then, in the final step, the same calculation as Step 5 in Figure 1 is conducted to get the relative 


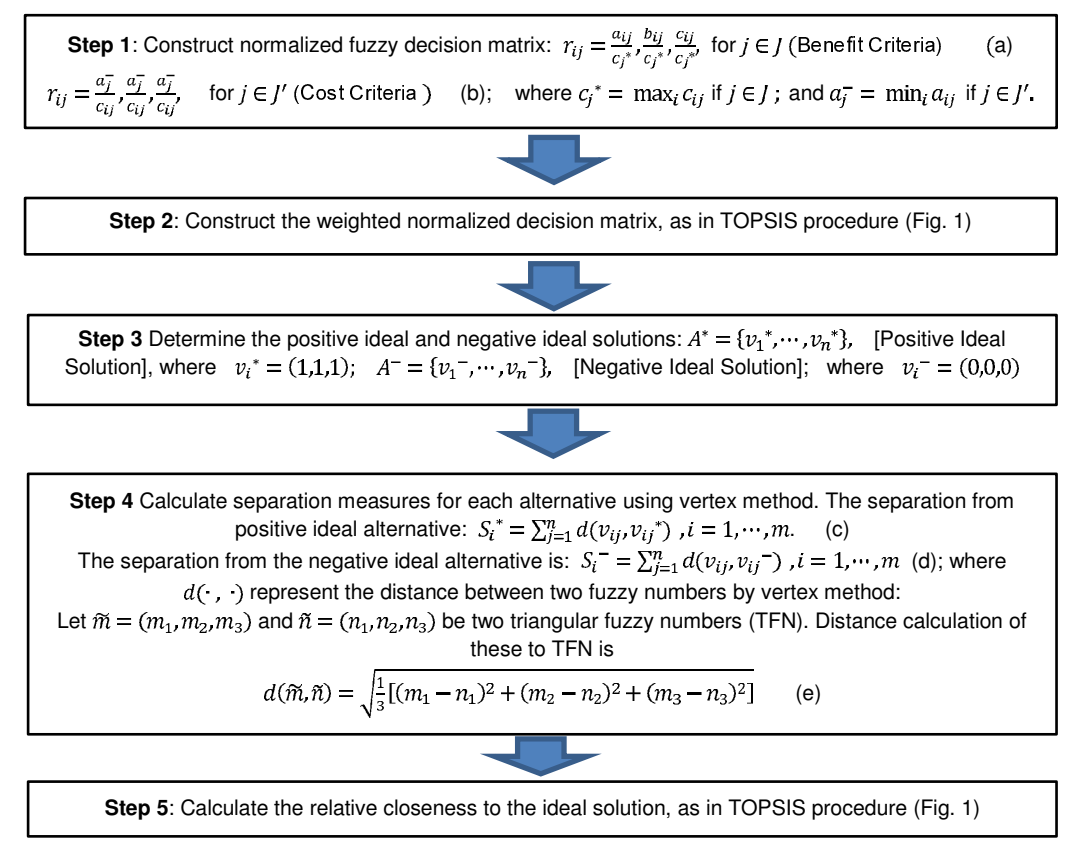

Fig. 2. Stepwise procedure of Fuzzy TOPSIS [4]

closeness to ideal solution $\left(C C_{i}\right)$. An alternative can now be ranked according to the descending order of $C C_{i}$ value.

\section{A. Analyzing the Steps of Fuzzy TOPSIS in a Practical Context}

In order to show how each stage in the FTOPSIS method shown in Figure 2 works, we provide a practical example taken from Chen [4]. We briefly explain step by step the FTOPSIS procedure which is applied in this practical example.

Suppose that a software company desires to hire a system analysis engineer. After preliminary screening, three candidates A1, A2 and A3 remain for further evaluation. A committee of three decision-makers, D1, D2 and D3 has been formed to conduct the interview and to select the most suitable candidate. Five benefit criteria are considered; emotional steadiness $(\mathrm{C} 1)$; oral communication skill $(\mathrm{C} 2)$, personality (C3), past experience (C4) and self-confidence (C5). The decision-makers use the linguistic weighting variables (shown in Table I) to assess the importance of the criteria and present it in Table II.

Following rating, the next step is to aggregate all the preferences values to form a fuzzy decision matrix using (1) and (2). For this example, the results of this process are shown in Table III. Post aggregation, a normalization step (Step 1 in Figure 2) is applied, where (a) is for the benefit criteria and (b) is for the cost criteria.

This step is taken to facilitate the computational problems inherent to the presence of the different units in the decision matrix and aims at obtaining comparable scales. In this example, it uses all the benefit criteria. Then, for the overall weighted normalized decision matrix, this method uses Step 2 shown in Figure 2. In this step, each value from the previous
TABLE I

LINGUISTIC SCALE FOR WEIGHTING OF CRITERIA AND RATING OF ALTERNATIVES IN FUZZY TOPSIS METHOD [4]

\begin{tabular}{|l|l|l|l|l|}
\hline \multicolumn{5}{|c|}{ (a) Linguistic scale for weight of criteria } \\
\hline Low (L) & $\begin{array}{l}\text { Medium } \\
\text { Low (ML) }\end{array}$ & $\begin{array}{l}\text { Medium } \\
(\mathrm{M})\end{array}$ & $\begin{array}{l}\text { Medium } \\
\text { High } \\
\text { (MH) }\end{array}$ & High (H) \\
\hline$(0,0.1,0.3)$ & $(0.1,0.3,0.5)$ & $(0.3,0.5,0.7)$ & $(0.5,0.7,0.9)$ & $(0.7,0.9,1.0)$ \\
\hline \multicolumn{5}{|c|}{ (b) Linguistic scale for rating of alternatives } \\
\hline Poor (P) & $\begin{array}{l}\text { Medium } \\
\text { Poor (MP) }\end{array}$ & Fair (F) & $\begin{array}{l}\text { Medium } \\
\text { Good } \\
\text { (MG) }\end{array}$ & Good (G) \\
\hline$(0,1,3)$ & $(1,3,5)$ & $(3,5,7)$ & $(5,7,9)$ & $(7,9,10)$ \\
\hline
\end{tabular}

TABLE II

LINGUISTIC RATING OF ALTERNATIVES AND IMPORTANCE WEIGHT OF CRITERIA

\begin{tabular}{|l|l|lll|}
\hline & & \multicolumn{3}{|c|}{ DM's rating } \\
\hline Crit. & Alts. & $D_{1}$ & $D_{2}$ & $D_{3}$ \\
\hline$C_{1}$ & $A_{1}$ & MG & $\mathrm{G}$ & $\mathrm{MG}$ \\
& $A_{2}$ & $\mathrm{G}$ & $\mathrm{G}$ & $\mathrm{MG}$ \\
& $A_{3}$ & MG & MG & $\mathrm{F}$ \\
\hline \multicolumn{2}{|c|}{ Weight } & H & VH & MH \\
\hline$C_{2}$ & $A_{1}$ & $\mathrm{G}$ & MG & $\mathrm{F}$ \\
& $A_{2}$ & $\mathrm{G}$ & $\mathrm{G}$ & $\mathrm{G}$ \\
& $A_{3}$ & MG & $\mathrm{G}$ & $\mathrm{G}$ \\
\hline \multicolumn{2}{|c|}{ Weight } & VH & VH & VH \\
\hline$C_{3}$ & $A_{1}$ & $\mathrm{~F}$ & $\mathrm{MG}$ & $\mathrm{MG}$ \\
& $A_{2}$ & $\mathrm{G}$ & $\mathrm{G}$ & $\mathrm{MG}$ \\
& $A_{3}$ & $\mathrm{G}$ & MG & MG \\
\hline \multicolumn{2}{|c|}{ Weight } & VH & H & H \\
\hline
\end{tabular}


TABLE III

FUZZY DECISION MATRIX

\begin{tabular}{|c|ccc|}
\hline & $C_{1}$ & $C_{2}$ & $C_{3}$ \\
\hline$A_{1}$ & $(5.67,7.67,9.33)$ & $(5.00,7.00,8.67)$ & $(5.67,6.33,8.33)$ \\
$A_{2}$ & $(6.33,8.33,9.67)$ & $(7.00,9.00,10.00)$ & $(6.33,8.33,9.67)$ \\
$A_{3}$ & $(4.33,6.33,8.33)$ & $(6.33,8.33,9.67)$ & $(5.67,7.67,9.33)$ \\
\hline Weight & $(0.70,0.90,1.00)$ & $(0.90,1.00,1.00)$ & $(0.77,0.93,1.00)$ \\
\hline
\end{tabular}

TABLE IV

WEIGHTED NORMALIZED DECISION MATRIX

\begin{tabular}{|c|ccc|}
\hline & $C_{1}$ & $C_{2}$ & $C_{3}$ \\
\hline$A_{1}$ & $(0.41,0.71,0.97)$ & $(0.45,0.70,0.87)$ & $(0.45,0.61,0.86)$ \\
$A_{2}$ & $(0.46,0.77,0.10)$ & $(0.63,0.90,1.00)$ & $(0.50,0.80,1.00)$ \\
$A_{3}$ & $(0.31,0.59,0.86)$ & $(0.57,0.83,0.97)$ & $(0.45,0.74,0.97)$ \\
\hline
\end{tabular}

normalized matrix is multiplied by its associated weight as shown in Table III.

The result of the weighted normalized decision making for this method are presented in Table IV. The next step is the definition of the ideal solution - in this case, the perfect value is used. For this example, the Fuzzy PIS is defined as in (3) while the Fuzzy NIS as in (4). These values, arising from Step 3 in Figure 2 by which the definition of this ideal solution is the perfect value for each criteria.

$$
\begin{aligned}
& A^{+}=[(1,1,1),(1,1,1),(1,1,1)] \\
& A^{+}=[(0,0,0),(0,0,0),(0,0,0)]
\end{aligned}
$$

The next step is to find the distance between the ideal solution and each of the alternatives. This method use the technique as shown in Step 4 in Figure 2 to establish this distance.

For this example, the distance of each alternative is shown in Table $\mathrm{V}$ together with the relative closeness $\left(C C_{i}\right)$ (Step 5) value and ranking of alternatives. In this real-world numerical example, alternative $\mathrm{A} 2$ is the best one followed by $\mathrm{A} 3$ and A1.

\section{B. Extensions of Basic Fuzzy TOPSIS (FTOPSIS)}

There are many extensions made in FTOPSIS method in order to produce supposedly better and more trustworthy results. Prior work by Chen used triangular fuzzy numbers to express evaluation of each alternative and weight of criterion by the linguistic terms. Chu [12] proposed to solve decision making problems by converting the fuzzy problem into a crisp form and solving it using standard TOPSIS. The proposed

TABLE V

DistanCE MEASUREMENTS, THE CLOSENESS COEFFICIENT AND THE RANK OF ALTERNATIVES IN FTOPSIS METHOD

\begin{tabular}{|c|cccc|}
\hline & dist $_{i}^{+}$ & dist $_{i}^{-}$ & $C C_{i}$ & Rank \\
\hline$A_{1}$ & 1.15 & 2.09 & 0.65 & 3 \\
$A_{2}$ & 0.87 & 2.43 & 0.74 & 1 \\
$A_{3}$ & 1.09 & 2.19 & 0.67 & 2 \\
\hline
\end{tabular}

method first develop the membership function for all ratings and weightings using interval arithmetic of fuzzy numbers, and then defuzzified them into crisp values using a ranking method. Tsaur et al. [13] changed a FTOPSIS problem into a crisp one via a centeroid method and then solved the non-fuzzy MADM problem using the standard TOPSIS method. Jahanshahloo [14] proposed an extended TOPSIS method by using the concept of $\alpha$-cuts. Wang and Lee [15] generalized the TOPSIS method in fuzzy data and proposed 'Up' and 'Lo' operations on fuzzy numbers to find the ideal solution and negative ideal solution. Mahdavi et al. [16] proposed a revised FTOPSIS method through the introduction of appropriate negations for obtaining ideal solutions and applied a new measurement of fuzzy distance value with a lower bound of alternatives. Chen and Lee [17] proposed an interval type-2 FTOPSIS method to better handle the imprecise and vagueness information which exist in multi attributes decision making problems.

\section{Limitations, Challenges AND Issues in FuZZy TOPSIS}

In this section, we identify the main limitations, issues and challenges in the existing FTOPSIS method since its introduction in 2000 by Chen [4]. We provide the list of basic characteristic of the FTOPSIS method in Table VI.

First of all, FTOPSIS use cardinal information on attributes in analysis. The main process in FTOPSIS is to determine the distance between fuzzy PIS and fuzzy NIS to each alternative respectively. This method falls in Multi Attribute Decision Making (MADM) category since attribute information is given. However, in existing FTOPSIS method, there are no consistency and reliability checks, as explained below. We believe that these two characteristics are very important in any decision making process as it may lead to misleading result. These can be considered as fundamental aspects that need to be handled to produce a better decision. We will discuss these issues in Section IV-A. The basic TOPSIS method can accommodate many attributes and alternatives at the same time and so it can be described as a 'compensatory operation' technique.

\section{A. What is Lacking in the Literature?}

Based on initial work on FTOPSIS by Chen [4] as described in Section III, the DMs judgement or preferences are described in fuzzy sets using triangular fuzzy numbers (TFN). Such a set is used in order to handle any linguistic uncertainty which may exist in the preferences of decision makers. In most FTOPSIS papers, this linguistic uncertainty is known as the 'fuzzy environment'. Most enhancements of FTOPSIS were made based on improving the capability in handling linguistic uncertainty. For example, instead of using ordinary fuzzy set information (type-1 fuzzy sets), some researchers attempted to use type-2 sets [18] in the belief that they better represent any linguistic uncertainties. Such uncertainty can be considered as fuzziness in information. However, realworld information is not only characterized by fuzziness. A subtly different characteristic in real-world information is 
TABLE VI

CHARACTERISTICS OF THE TOPSIS METHOD

\begin{tabular}{|c|l|l|}
\hline No. & Characteristics & TOPSIS \\
\hline 1 & Category & $\begin{array}{l}\text { Cardinal information, in- } \\
\text { formation on attributes, } \\
\text { MADM }\end{array}$ \\
\hline 2 & Core process & $\begin{array}{l}\text { The distances from PIS } \\
\text { and NIS(cardinal absolute } \\
\text { measurement) }\end{array}$ \\
\hline 3 & Attribute & Given \\
\hline 4 & Weight elicitation & Given \\
\hline 5 & Consistency check & None \\
\hline 6 & Reliability check & None \\
\hline 7 & $\begin{array}{l}\text { No. of attributes ac- } \\
\text { commodated }\end{array}$ & Many more \\
\hline 8 & $\begin{array}{l}\text { No. of alternatives ac- } \\
\text { commodated }\end{array}$ & Many more \\
\hline 9 & Others & Compensatory operation \\
\hline
\end{tabular}

that of 'partial reliability' [19]. Thus, it is not adequate to use only fuzzy numbers in handling fuzziness, but there is also a need for other information that can formally describe the partial reliability characteristic in real-world information. For example, in Section III-A, DMs provide a rating as Good without stating the confidence level in the source of information. In literature, some researchers have proposed tools to assess reliability of information. For example, the concept of fuzzy measures [20] and fuzzy integrals [21]. A practical application of fuzzy measures and fuzzy integrals can be seen in [22], which deals with partially reliable data in a crowd-sourcing context. Recently, Zadeh introduced a new concept which he termed the 'Z-number' [23] to deal with reliability of information. It is an ordered pair $Z=(A, B)$ of fuzzy numbers used to describe a value of variable $X$, where $A$ is an imprecise constraint on values of $X$, and $B$ is an imprecise estimation of reliability of $A$, which can be viewed as a value of probability measure of $A$ [19]. Further details of reliability and Z-numbers can be found in [19] and [23].

It is absolutely clear that various uncertainties other than linguistic uncertainty exist in FTOPSIS problems. As an example, there may exist uncertainty due to missing information. For example, in the numerical example presented in Section III-A, decision makers naturally do not provide ratings with a certain numerical value (i.e., crisp values) but would rather provide a prediction such as: "The rating of Candidate 1 (A1) with respect to Criteria 1 (C1) is Medium Poor." It can be assumed that this DM is certainly sure about his/her preferences. Consider one more example given by a DM: "The rating of Candidate 1 (A1) with respect to Criteria 1 (C1), probably is Medium Poor." In this sentence, the DM seems unsure about the given rating in this particular event, by explicit use of the word probably in the preference statement. Thus, in order to take such situations into account, the concept of restriction should be used together in describing realworld information. It is suggested in [24] to use possibility distributions to represent the state of events in imprecise realworld information. However, the probability distribution of an event also needs to be considered. This will indicate to what degree does the Medium Poor rating in this example fit the DM's perception about Medium Poor.

Based on the application of FTOPSIS in the example shown in Section III-A, the rating process has constructed from a sentence that is known as a 'proposition' in natural language. When fuzzy information is used, the proposition is now known as a fuzzy proposition. The natural language used in the fuzzy proposition may contain rating levels, known as fuzzy predicates (e.g., Good, Poor), and/or quantifiers (e.g., Very). Zadeh [25] highlighted that the truth and meaning of a proposition drawn from a natural language may be represented as a restriction. A restriction, $R$ on variable $X$, or $R(X)$, may be viewed as an answer to a question of the form: What is the rating for $X$ ? Thus, the variable $X$ must be clear in order to answer the question. What really matters is not understanding of meaning the variable $X$, but precisiation of meaning. This is Zadeh's method of representation which has a formal mathematical definition which can then be accurately used in computation. Precisiation of meaning is a prerequisite to reasoning and computation with information described in natural language [25]. Thus, we believe in relation to the reliability aspect of information, it is important to take into account the truth and meaning of a proposition which can be described as a restriction of the existing FTOPSIS method. This can be done in an initial step of FTOPSIS method prior to carrying out the usual Step 1. The meaning concept in FTOPSIS can be implemented in situations in which the DMs faced uncertainty in determining the rating for each alternative. The meaning of rating in FTOPSIS method can be achieved by a precisiation step. The detail of this step is not discussed here due to limited space. Please refer to [25] for more detail.

In the concept of truth degree, there is a close relationship with the concept of meaning, as described in the previous paragraph. To measure the truth value of any proposition in natural language, it is necessary to understand the meaning of the proposition. As we stated in previous paragraph, to assess the meaning of proposition, we need to precisiate the proposition. For example, in the FTOPSIS numerical application in Section III-A, a rating proposition may exist as "The rating of A1 with respect to $\mathrm{C} 1$ is Certainly Good". In this proposition, the information consist of three relations: Good [rating, $\mu$ ], Certainly [proportion, $\mu$ ], and Population [A1 with respect to $\mathrm{C} 1$, rating].

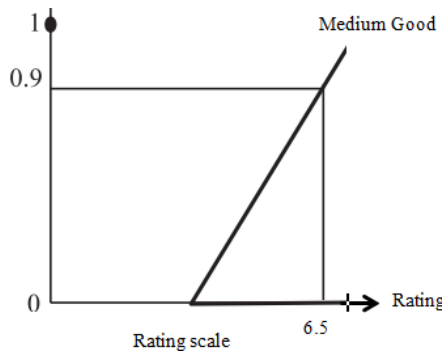

Fig. 3. Example of truth degree 
In this example, Good can have a value $\mu_{1}$ which indicates the grade of membership of rating in rating scale while certainly can have a value $\mu_{2}$ which indicates a grade of membership of level of confidence that the DM feels fits his/her perception. The overall meaning of that proposition can be described as the possibility distribution associated with the fuzzy set Good. Informally, it is the possibility that A1 is Good, and is equal to the grade of membership of A1 in Good. In the FTOPSIS application example in Section III-A, a rating from $\mathrm{DM}_{1}$ for $\mathrm{A} 1$ with respect to $\mathrm{C} 1$ is Medium Good which can have the degree of truth, $\mu_{\text {truth }}=0.9$. Assuming that the precisiation step has already been taken, then the resultant meaning is the value of 6.5 . Then, the truth degree can be assessed, calculated as 0.9 and shown graphically as in Figure 3. From this truth value, it is expected that the level of confidence for each DM can be measured and will lead to a reliable decision in the FTOPSIS method. This process can be implemented in an initial step of a FTOPSIS method which preceeds the usual Step 1.

Overall, we can summarize that reliability, truth and meaning issues are not considered sufficiently in existing FTOPSIS methodology. In the next section, we will further explore briefly one issue only, that of reliability, due to limited space. We will include the other issues to be discussed in our future work.

\section{B. Reliability of Information in Fuzzy TOPSIS}

Reliability of information can be viewed as to which extent we can rely on the source of the information and therefore the information itself. In other words, any approximation of values of interest, whether precise or not, is dependent on the confidence in the sources of information we deal with. Reliable information is dependable, trustworthy, unfailing, sure, authentic, genuine and reputable. Furthermore, a question may arise in real-world problem as "How reliable are the numbers which we deal with?".

Thus, in decision making process, the reputation of the sources of information is critical. As in FTOPSIS method, all information is collected from humans as decision makers. The partial reliable characteristic in real-world information has meant that the process of decision making based on FTOPSIS is a very challenging task. Therefore, in the next section, we will suggest a potential solution which could be used in future to achieve reliable decision.

\section{A Potential Solution}

In this section, with regard to the reliability issue, we now propose a potential solution which can address the imprecision and (un-)reliability of information at the same time. Please note that only the original fuzzy TOPSIS method introduced by Chen will be used as a base method. The proposed framework is shown in Figure 4.

The proposed framework starts with an initial step in which the decision maker gives a rating for each alternative with respect to each criterion. Suppose there is one decision maker evaluating $k$ alternatives with respect to $m$ criteria. Let the decision matrix $\mathbf{D}=d_{i j}$, where $d_{i j}$ is a Z-number, consisting of two fuzzy numbers, one for rating and one for reliability of the decision maker, and where $i=1, \cdots, k, j=1, \cdots, m$. In this step, the information is in the form of fuzzy sets. Then, the same procedure is taken as shown in Step 1 (Figure 4). In Step 2, the weighted normalized fuzzy decision matrix is constructed by multiplying the previous values in the decision matrix with the associated weight given by the decision maker. Prior to step 3, we propose to transform all the fuzzy set information for rating and reliability into crisp numbers. This step is needed in order to measure the distance between ideal solution points and each alternative. By doing this, we get the accurate distance since we applied the $n$-Euclidean distance formula with the crisp number. Please note that, this distance formula is impractical for fuzzy set values since the meaning of the fuzzy set information is different, as compared to crisp numbers. It should be noted that, the common practice in the fuzzy TOPSIS field is to treat fuzzy set information as a crisp number. Therefore, this may lead to significant loss of information and misleading results. Next, the following steps 4 and 5 are carried out, as in the standard TOPSIS method as shown in Figures 4 and 1. The result will be better in terms of reliability, accuracy and trustworthiness of the decision.

\section{Conclusion}

In this paper, we have briefly discussed the key stages involved in both the standard TOPSIS introduced by Hwang and Yoon, and the Fuzzy TOPSIS method introduced by Chen, carefully detailing each step. We believe this is the first work which has attempted to both provide details and to highlight the key differences between these two methods in a systematic manner. In addition, we have identified and discussed the limitations, issues and challenges which we believe have not been investigated sufficiently in the context of Fuzzy TOPSIS. Then, we have focussed on one main issue, that of reliability, which we have discussed further. Following identification and clarification of this issue, we have provided a potential solution which we believe could result in better and more trustworthy decisions. However, we are not able to provide numerical experimentation of the proposed new framework due to limited space. In addition, the aim of this paper is to provide the details of the TOPSIS and Fuzzy TOPSIS methods as well as their issues, limitations and changes and to provide a novel contribution on comparison between these two methods in systematic stepwise procedure. In the future, we will present and show the practical application of this proposed framework, and present further details on how this improves the existing fuzzy TOPSIS methods by providing more reliable and trustworthy decisions.

\section{ACKNOWLEDGMENT}

This paper was funded in part by the EPSRCs Neodemographics: Opening Developing World Markets by Using Personal Data and Collaboration grant, EP/L021080/1. 


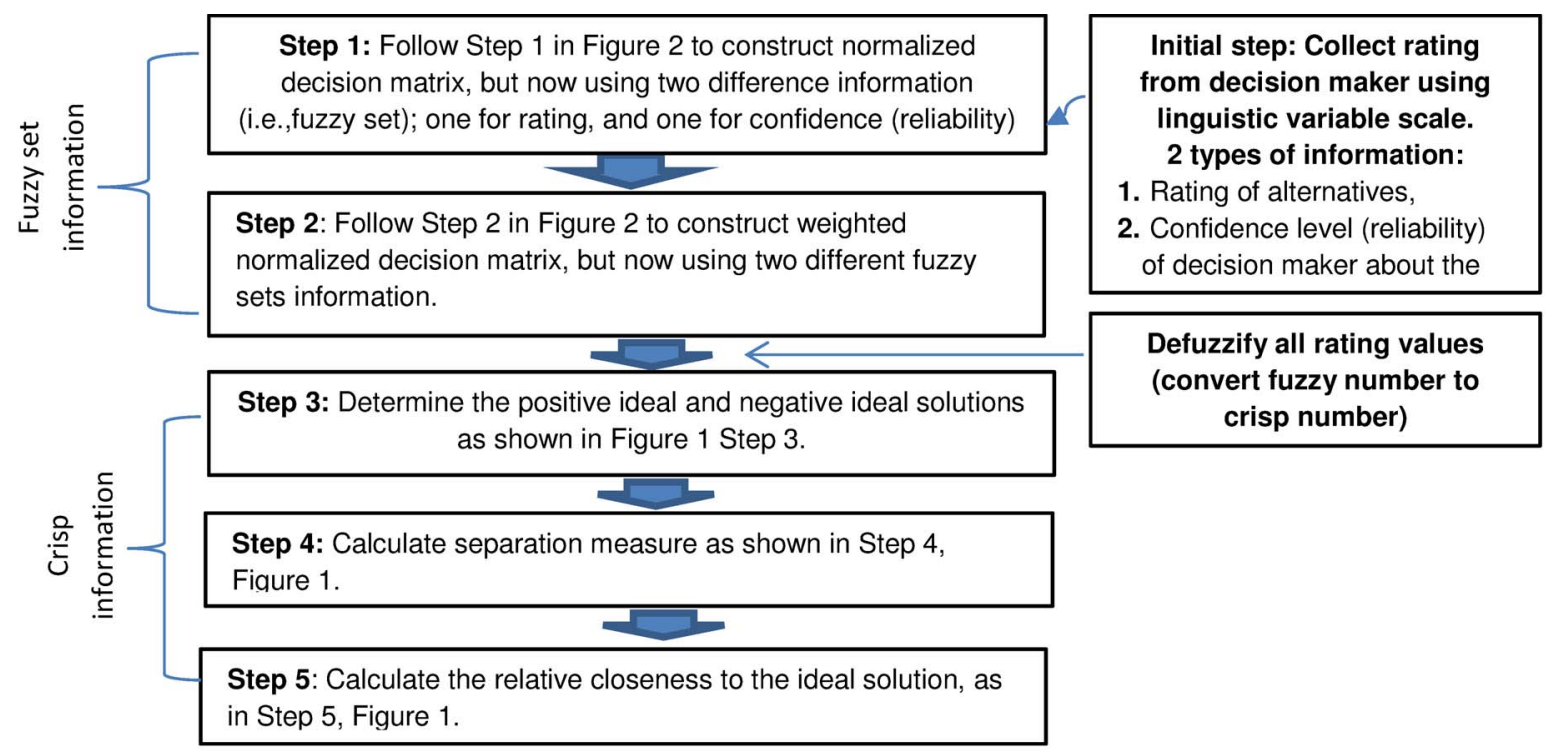

Fig. 4. Proposed new framework for Z-number based Fuzzy TOPSIS

\section{REFERENCES}

[1] C.-L. Hwang and K. Yoon, Multiple Attribute Decision Making : Methods and Application- A State of the Art Survey, lecture no ed., M. Beckmann and H. P. Kunzi, Eds. Springer Berlin Heidelberg New York, 1981.

[2] L. Zadeh, "Fuzzy Sets," Inf. Control, vol. 8, pp. 338-353, 1965.

[3] M. Behzadian, S. Khanmohammadi Otaghsara, M. Yazdani, and J. Ignatius, "A state-of the-art survey of TOPSIS applications," Expert Syst. Appl., vol. 39, pp. 13 051-13069, dec 2012.

[4] C.-T. Chen, "Extensions of the TOPSIS for group decision-making under fuzzy environment," Fuzzy Sets Syst., vol. 114, no. 1, pp. 1-9, aug 2000.

[5] S. M. Chen and L. W. Lee, "Fuzzy multiple attributes group decisionmaking based on the interval type-2 TOPSIS method," Expert Syst. Appl., vol. 37, no. 4, pp. 2790-2798, 2010.

[6] F. E. Boran, S. Genç, M. Kurt, and D. Akay, "A multi-criteria intuitionistic fuzzy group decision making for supplier selection with TOPSIS method," Expert Syst. Appl., vol. 36, no. 8, pp. 11 363-11 368, oct 2009.

[7] T. Y. Chen and C. Y. Tsao, "The interval-valued fuzzy TOPSIS method and experimental analysis," Fuzzy Sets Syst., vol. 159, pp. 1410-1428, 2008.

[8] C. Kahraman, Fuzzy Multi-Criteria Decision Making, springer o ed., P. M. Pardalos and D.-Z. Du, Eds. Springer Science+Business Media, LLC, 2008.

[9] S.-J. Chen and C.-L. Hwang, Fuzzy Multiple Attribute Decision MakingMethods and Applications. Springer-Verlag, 1992.

[10] I. Ertugrul and N. Karakasoglu, "Comparison of fuzzy AHP and fuzzy TOPSIS methods for facility location selection," Int. J. Adv. Manuf. Technol., vol. 39, pp. 783-795, 2007.

[11] F. R. Lima Junior, L. Osiro, and L. C. R. Carpinetti, "A comparison between Fuzzy AHP and Fuzzy TOPSIS methods to supplier selection," Appl. Soft Comput., vol. 21, pp. 194-209, 2014.

[12] T. C. Chu, "Selecting plant location via a fuzzy TOPSIS approach," Int. J. Adv. Manuf. Technol., vol. 20, no. 11, pp. 859-864, 2002.

[13] S.-H. Tsaur, T.-Y. Chang, and C.-H. Yen, "The evaluation of airline service quality by fuzzy MCDM," Tour. Manag., vol. 23, pp. 107-115, 2002.

[14] G. R. Jahanshahloo, F. H. Lotfi, and M. Izadikhah, "Extension of the TOPSIS method for decision-making problems with fuzzy data," Appl. Math. Comput., vol. 181, pp. 1544-1551, 2006.

[15] Y.-J. Wang and H.-S. Lee, "Generalizing TOPSIS for fuzzy multiple-criteria group decision-making," Comput. Math. with Appl., vol. 53, no. 11, pp. 1762-1772, jun 2007. [Online]. Available: http://linkinghub.elsevier.com/retrieve/pii/S0898122107001514
[16] I. Mahdavi, N. Mahdavi-Amiri, A. Heidarzade, and R. Nourifar, "Designing a model of fuzzy TOPSIS in multiple criteria decision making," Appl. Math. Comput., vol. 206, no. 2, pp. 607-617, 2008. [Online]. Available: http://dx.doi.org/10.1016/j.amc.2008.05.047

[17] S.-M. Chen and L.-W. Lee, "Fuzzy multiple attributes group decisionmaking based on the interval type-2 TOPSIS method," vol. 37, no. 4, pp. $2790-2798,2010$.

[18] L. Zadeh, "The concept of a linguistic variable and its application to approximate reasoningPart I," Inf. Sci. (Ny)., vol. 8, pp. 199-249, jan 1975.

[19] R. A. Aliev, O. H. Huseynov, R. R. Aliyez, and A. A. Alizadeh, The Arithmetic of Z-Numbers : Theory and Applications, 2015.

[20] Z. Wang and G. J. Klir, Fuzzy Measure Theory. Springer Science+Business Media New York, 1992.

[21] M. Sugeno, "Theory of fuzzy integrals and its applications," Ph.D. dissertation, Tokyo Institute of Technology, 1974.

[22] C. Wagner and D. T. Anderson, "Extracting meta-measures from data for fuzzy aggregation of crowd sourced information," in 2012 IEEE Int Conf. Fuzzy Syst., 2012, pp. 1-8.

[23] L. Zadeh, "A Note on Z-numbers," Inf. Sci. (Ny)., vol. 181, no. 14, pp. 2923-2932, jul 2011.

[24] D. Dubois, "The role of fuzzy sets in decision sciences: Old techniques and new directions," Fuzzy Sets Syst., vol. 184, no. 1, pp. 3-28, 2011.

[25] L. Zadeh, "Toward a restriction-centered theory of truth and meaning (RCT)," Inf. Sci. (Ny)., vol. 248, pp. 1-14, 2013. 